\title{
PROPUESTA DE MODELO DE UNIVERSIDAD CORPORATIVA
}

\section{PROPOSED MODEL OF CORPORATE UNIVERSITY}

\author{
Manuel Alfonso Garzón Castrillon \\ Grupo de Investigación FIDEE \\ Fundación para la Investigación y el Desarrollo Educativo Empresarial \\ Barranquilla, Colombia, Sur América \\ manuelalfonsogarzon@fidee.org
}

Fecha de recepción: 06/06/2018 - Fecha de aprobación: 15/08/2018

\section{RESUMEN}

En este artículo de revisión, se abordan en primer lugar los antecedentes, buscando identificar cuáles fueron las primeras universidades corporativas, posteriormente se hace una aproximación a la definición del concepto de universidad corporativa y se propone la definición que orientará el documento; como resultado de la revisión bibliográfica se elabora la propuesta de un modelo de Universidad Corporativa, que muestra la relación entre cuatro procesos de la universidad corporativa clave, la dirección y el compromiso de la alta dirección; la gestión del conocimiento y el aprendizaje organizacional; la gestión humana; y las tecnologías para la gestión del conocimiento, y sus variables muestran la relación entre cuatro procesos claves de la universidad corporativa: la dirección y el compromiso de la alta dirección; la gestión del conocimiento y el aprendizaje organizacional; la gestión humana; y las tecnologías para la gestión del conocimiento, finalmente se elaboran unas conclusiones, entre las que se destacan que las universidades corporativas deben hacer frente a nuevas misiones, tales como la elaboración de programas con los clientes internos, acompañamiento individual y de equipo, consejería y coaching de proyectos y acompañamiento del cambio, o también la gestión de proceso de innovación con los clientes.

PALABRAS CLAVE: Universidades Corporativas; Gestión del Conocimiento; Aprendizaje Organizacional; Gestión Humana.

\section{ABSTRACT}

In this review article, we first address the antecedents, trying to identify which were the first corporate universities, then an approach to the definition of the concept of corporate university is proposed and the definition that will orient the document, result of the revision a proposal for a model of Corporate University shows the relationship between four processes of the key corporate university, the direction and commitment of top management; knowledge management and organizational learning; human management; and technologies for knowledge management, and their variables show the relationship between four key processes of the corporate university: the direction and "Visión de Futuro" Año 16, Volumen No 23 N¹, Enero - Junio 2019 - Pág. 1 - 23

URL de la Revista: http://revistacientifica.fce.unam.edu.ar/

URL del Documento: http://revistacientifica.fce.unam.edu.ar/index.php?option=com content\&view=article\&id=492\&Itemid=101 
commitment of senior management; knowledge management and organizational learning; human management; and technologies for the management of knowledge, finely elaborate conclusions, among which it is emphasized that Corporate universities must face new missions; such as the elaboration of programs with internal clients, individual and team accompaniment, advice and project coaching and accompaniment of the change, or also the process management of innovation with the clients.

KEYWORDS: Corporate Universities; Knowledge Management; Organizational Learning; Human Management.

\section{INTRODUCCIÓN}

La revisión de la literatura revela la falta de evidencia empírica sobre cómo una Universidad Corporativa (UC) debe conceptualizar su operación como una función corporativa efectiva para respaldar los objetivos de la organización. La mayoría de las publicaciones relacionadas con UC se centran en uno o dos aspectos de la operación de UC, algunos investigadores se centran en las estrategias de aprendizaje y el gobierno de UC como Rademakers, 2005; Rivera y Paradise, 2006, mientras que otros examinan los métodos de evaluación, como Allen, 2002; Bober \& Bartlett, 2004 y otros incluyen el desarrollo de gerentes y líderes como Storey, (2004) o las fuentes de financiación e implementación de tecnología, Anderson, 2003; Macpherson, Homan y Wilkinson, 2005, y alianzas con otras funciones, internas o externas a la firma, Blass, 2005; Thompson, 2000. Hay un número limitado de estudios exhaustivos de operaciones de UC y la mayoría usa uno o pocos estudios de casos, por ejemplo, Baldwin, Danielson y Wiggenhorn, 1997; El-Tannir, 2002; Holland \& Pyman, 2006; Jansink, Kwakman, \& Streumer, 2005; Shaw, 2005; por su parte Allen, 2002, Meister, 1998 afirman que las prácticas de UC consisten en un número limitado de estudios de casos o mejores prácticas y a menudo carecen de fundamento teórico o conceptual.

En este artículo de revisión, se abordan en primer lugar los antecedentes, buscando aproximarnos a identificar las primeras universidades corporativas, posteriormente se hace una acercamiento a la definición del concepto de Universidad Corporativa y se propone la definición que orientará el documento; el siguiente tema es el de la propuesta de un modelo de Universidad Corporativa y sus variables, que muestra la relación entre cuatro procesos claves de la Universidad Corporativa: la dirección y el compromiso de la alta dirección; la gestión del conocimiento y el aprendizaje organizacional; la gestión humana; y las tecnologías para la gestión del conocimiento, finalmente se elaboran unas conclusiones.

\footnotetext{
"Visión de Futuro" Año 16, Volumen No 23 N¹, Enero - Junio 2019 - Pág. 1 - 23

URL de la Revista: http://revistacientifica.fce.unam.edu.ar/

URL del Documento: http://revistacientifica.fce.unam.edu.ar/index.php?option=com content\&view=article\&id=492\&Itemid=101

ISSN 1668 - 8708 - Versión en Línea

E-mail: revistacientifica@fce.unam.edu.ar
} 


\section{DESARROLLO}

\section{Antecedentes}

Según Young, C.; Tuttle, R. (1969), la noción de Universidad Corporativa aparece por primera vez antes de la segunda guerra mundial en General Motors en Flint, estado de Michigan, Estados Unidos en 1919. La idea de los dirigentes de General Motors era poder estructurar a la vez los procesos de fabricación de la empresa y hacer estos conocimientos transmisibles, pero también construir una reputación de excelencia alrededor de los saber-hacer de la empresa, por tanto, la General Motors Institute llegó a ser una Universidad Corporativa.

De esta manera para Anderson, (2000) la Universidad Corporativa (UC) es un modelo emergente de capacitación continua en el mundo corporativo y aprendizaje continuo para los empleados. La primera Universidad Corporativa pionera fue la de General Electric en 1950 y, más recientemente, ese modelo para la capacitación de empleados ha sido adoptado por un gran número de corporaciones en todo el mundo. Según las estadísticas actualizadas, había 400 Universidades Corporativas en los Estados Unidos a comienzos de la década de 1990 y ese número había crecido a casi 1800 a fines del 2000.

Según Nell Eurich (1985) en 1985, los Estados Unidos contaba con dieciocho Corporate Colleges o Universidades Corporativas, este es el primer estudio exhaustivo en ese país y con el paso del tiempo el número aumenta considerablemente hasta alcanzar 3700 para el año 2010. De esta manera la mayoría de los grandes grupos empresariales, en Europa, en Asia o en los Estados Unidos, tienen su propia Universidad Corporativa. Aunque una de las más importantes es la de Accenture a Saint-Charles (estado de Indiana) con un inmenso campus (2000 habitaciones, 700 personas a tiempo pleno) y sus 60000 participantes anuales, la de mayor reputación es la de General Electric.

En 1961, afirma Rees, G.; y Smith P. (2014), es el gigante de la comida rápida, McDonald, quien ha empleado el término de Universidad Corporativa en la creación de Hamburger University, por tanto, hace mucho tiempo que los americanos han elegido el término universidad para hablar de sus propias estructuras educativas internas, las corporate universities. Para el año 2018, se cuentan aproximadamente con 4000 universidades alrededor del mundo.

En 1972, según Garbellini (2013), Fiat, como figura precursora en Europa, creó su Universidad Corporativa: ISVOR (Instituto SViluppo ORganisational). EI ISVOR ha contado en su seno aproximadamente con 150 profesores a tiempo completo.

En la Tabla No1, se muestra una relación no exhaustiva del inicio de labores de algunas Universidades Corporativas:

Tabla $N^{\circ} 1$. Inicio de labores de Universidades Corp orativas

\begin{tabular}{|c|l|c|}
\hline Año & \multicolumn{1}{|c|}{ Universidad Corporativa } & Autor(es) \\
\hline 1919 & General Motors Institute & Young, C.; Tuttle, R. (1969) \\
\hline 1950 & Universidad corporativa General Electric USA & Anderson, (2000), Ricardo (2007, p. 14) \\
\hline 1963 & Hamburger University of McDonalds USA & Rees, G.; y Smith P. (2014) \\
\hline
\end{tabular}

"Visión de Futuro" Año 16, Volumen N 23 N¹, Enero - Junio 2019 - Pág. 1 - 23

URL de la Revista: http://revistacientifica.fce.unam.edu.ar/

URL del Documento: http://revistacientifica.fce.unam.edu.ar/index. php?option=com content\&view=article\&id=492\&/temid=101

ISSN 1668 - 8708 - Versión en Línea

E-mail: revistacientifica@fce.unam.edu.ar 


\begin{tabular}{|c|c|c|}
\hline Año & Universidad Corporativa & Autor(es) \\
\hline 1972 & $\begin{array}{l}\text { Universidad Corporativa: ISVOR (Instituto SViluppo } \\
\text { ORganisational) FIAT, Italia }\end{array}$ & Walton (1999; Garbellini (2013) \\
\hline 1979 & Universidad Corporativa Gamesa Mx & Viscaña P y Uri be E. (2014) \\
\hline 1982 & Motorola USA & Viscaña P y Uri be E. (2014) \\
\hline 1992 & Grupo Accor Brasil & Eboli, (2004) \\
\hline 1993 & Oracle University USA & http://education.oracle.com/ \\
\hline 1996 & $\begin{array}{l}\text { Universidad Corporativa Hospital San Vicente de } \\
\text { Paul, Colombia }\end{array}$ & https://educacionsanvicentefundacion.com \\
\hline 1996 & Disney University (Disney Institute) & $\begin{array}{l}\text { Lipt, D. (2013) } \\
\end{array}$ \\
\hline 1997 & Cisco Networking Academy USA & $\begin{array}{l}\text { https://www.netacad.com/es/about-networking- } \\
\text { academy }\end{array}$ \\
\hline 2000 & Universidad Corporativa Fenosa Es. & Andreu R. (2001) \\
\hline 2000 & Liverpool México & Gómez (2018) \\
\hline 2000 & Jet Blue USA Florida & Viscaña P y Uri be E. (2014) \\
\hline 2003 & Pacific Rubiales Colombia & Viscaña P y Uri be E. (2014) \\
\hline 2004 & EPM Colombia & Viscaña P y Uri be E. (2014) \\
\hline 2005 & Tenaris University, Argentina & Aguirre et al (2017) \\
\hline 2006 & Microsoft IT Academy USA & Scott $(2006)$ \\
\hline 2008 & ECOPETROL Colombia & Viscaña P y Uri be E. (2014) \\
\hline 2008 & Universidad Arcor Argentina & www.universidad.arcor.com \\
\hline 2012 & Universidad de Telecom Argentina & Aguirre et al (2017) \\
\hline 2013 & Universidad Corporativa Bridgestone & Aguirre et al (2017) \\
\hline
\end{tabular}

Fuente: Elaboración Propia en base a los autores citados (2018)

Es importante resaltar la creciente sofisticación de las universidades corporativas que según Walton (1999) sugiere, un modelo de desarrollo de las universidades corporativas de primera, segunda y tercera generación debe centrarse tanto en el propósito como en la estrategia de aprendizaje. Walton utiliza la Universidad de Disney como un ejemplo típico de un tipo de primera generación, con un enfoque estrecho en la adopción de la cultura y los valores de la organización y principalmente las actividades basadas en el aula. Citando a Motorola como un ejemplo de una universidad de segunda generación, Walton (1999) sugiere que generalmente ofrecen una gama más amplia de actividades, a un rango de niveles dentro de la organización y pueden organizarse en áreas curriculares para abordar habilidades funcionales, problemas culturales y aprendizaje correctivo.

Este tipo de institución a menudo se caracteriza por asociaciones con otros empleadores, instituciones educativas y la comunidad en general. Las universidades corporativas de tercera generación, argumenta Walton (1999), son aquellas que buscan hacer el mejor uso de la nueva tecnología para el aprendizaje, y se caracterizan por el proceso en lugar del lugar, adoptando la estructura de una organización virtual. Phillips (1999) señala que esto es a menudo una característica de las universidades corporativas dentro del Reino Unido, que se desarrollaron más tarde que las instituciones estadounidenses y están mejor posicionadas para aprovechar los avances de la tecnología. La Universidad Corporativa de tercera generación es vista como el motor intelectual de la organización, desarrollando el capital humano de todos los empleados con un enfoque en el desarrollo de la creatividad y la innovación e impulsando el cambio estratégico.

Si bien la retórica de la universidad corporativa se basa en las agendas de aprendizaje y estrategia, también vale la pena señalar que algunos comentaristas han sugerido que el desarrollo de las universidades corporativas también es un intento de rediseñar los procesos comerciales para obtener el mejor valor. En consecuencia, representan no solo una apreciación corporativa renovada

\footnotetext{
"Visión de Futuro" Año 16, Volumen No 23 N¹, Enero - Junio 2019 - Pág. 1 - 23

URL de la Revista: http://revistacientifica.fce.unam.edu.ar/

URL del Documento: http://revistacientifica.fce.unam.edu.ar/index.php?option=com content\&view=article\&id=492\&Itemid=101

ISSN 1668 - 8708 - Versión en Línea

E-mail: revistacientifica@fce.unam.edu.ar
} 
para la educación, sino también "el deseo de centralizar los recursos para reducir los gastos" (Arnone, 1998, p. 200). Uno de los objetivos clave del proceso es la rentabilidad y "el cliente más importante de una universidad exitosa es el jefe de una unidad de negocios, no los participantes" (Arnone, 1998, p. 200). El objetivo de la capacitación debe ser asegurar que la capacitación no solo agregue valor al recurso humano, sino que el valor agregado también sea beneficioso para el empleador, que paga las facturas de capacitación.

De hecho, autores como Meister, (1997); Peak, (1997); Arnone, (1998); y Stumpf, (1998) coinciden en que este debe ser un objetivo clave, y perderlo socavaría la relación entre el negocio y el entrenamiento. Este impulso a la rentabilidad es a menudo en donde la nueva tecnología y el aprendizaje se convierten en un papel fundamental. Es costo-efectivo ofrecer a los aprendices las herramientas y la tecnología para continuar el proceso de aprendizaje en su trabajo y en su entorno social. Como afirma Arkin (2000, p. 43) "la repercusión de la tecnología, que está reduciendo el costo de impartir algunos tipos de capacitación, es una de las fuerzas impulsoras detrás del creciente interés en universidades corporativas". Aunque el e-learning presenta una ruta para lograr esto, el potencial de los sistemas tecnológicos está mediado por la forma en que se les da forma en el uso, así como por las capacidades y características de la tecnología (Dawson et al., 2003).

Por lo tanto, el impacto del e-learning dependerá de cómo se adopte y use la tecnología dentro de los contextos organizacionales y qué tan bien la tecnología respalda los objetivos, estrategias y valores del aprendizaje dentro del marco de la Universidad Corporativa. Las universidades corporativas apuntan a promover el aprendizaje y una cultura rica en conocimiento en todos los niveles dentro de la organización, a la vez que ubican el aprendizaje firmemente dentro del contexto y las necesidades organizacionales (Prince y Stewart, 2002). La utilización de las tecnologías de aprendizaje y comunicación en la creación de comunidades de aprendizaje locales, nacionales o mundiales es parte del panorama emergente de la Universidad Corporativa.

Aunque a finales de los noventa las Escuelas de Negocio consideraban las universidades corporativas como competidores (Lorange, P., 2002), ahora han entendido que vale más pensar en términos de cooperación y colaboración en vez de competencia, y cooperan, frecuentemente, con la elaboración y al desarrollo de los programas de estas universidades. El documento del IMD, International Institute for Management Development, (Strebel, P., 2005), que constituye una referencia sobre los posibles aportes de las Escuelas de Negocio a las universidades corporativas, explica por qué las primeras entienden tan claramente las necesidades de sus colegas.

Por lo tanto, siguiendo a Dealtry (2005), la razón de ser de la Universidad Corporativa surgió después de un largo período de crisis de identidad, esencialmente como agente animador y de cambio sistémico, introduciendo y ayudando en la evolución y gestión de nuevas formas de pensamiento y en la creación de muchos procesos de adaptación continua, que son necesarios para que la organización competitiva prospere y sobreviva a la dinámica del entorno en tiempo real.

Más allá de la puerta de aprendizaje de la administración, plantea Dealtry (2005) que hay posiblemente un tercer modelo educativo manifestándose, que puede contener elementos del modelo 
1, el modelo educativo tradicional, y el modelo 2, el modelo tradicional de capacitación y desarrollo de la empresa. (Ver figura $\mathrm{N}^{\circ} 1$ ). El objetivo es es tablecer una nueva perspectiva sobre el modelo 3. ¿Sería muy similar a los modelos 1 y 2 o alternativamente sería muy diferenciado? Hay muchas preguntas que abordar sobre los roles de los modelos 1 y 2 en el contexto de la intervención universitaria corporativa, por lo que es importante aumentar nuestro nivel de comprensión sobre las posibles conexiones y sinergias entre ellos antes de avanzar a través de la puerta de aprendizaje de la administración.

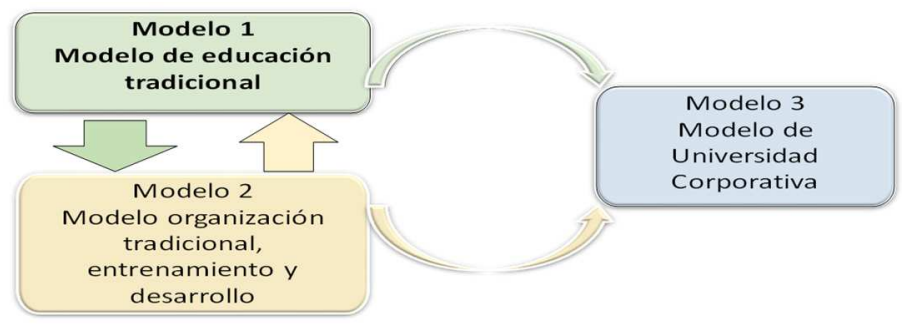

Figura N1. Surgimiento del modelo de Universidad C orporativa Fuente: Dealtry, R. (2005)

\section{Aproximación a la definición del concepto de Universidad Corporativa}

Hasta mediados de la década de 1990, para la mayoría de las organizaciones, las universidades corporativas se percibían como un fenómeno particularmente norteamericano. Se los consideraba departamentos de capacitación con distintivos, incluso con escepticismo, tanto por los especialistas en DRH como por los académicos por igual (Walton 1999). Aunque esta posición puede haber sido cierta en el pasado, hoy la situación ha cambiado drásticamente, ya que cada vez más organizaciones están haciendo serios intentos para crear universidades corporativas que manejen una variedad de necesidades individuales de aprendizaje y requisitos de desarrollo organizacional.

Investigadores como Lester (1999) han resaltado las dificultades para definir la Universidad Corporativa y haciendo alusión al diccionario de Oxford, define el término universidad como: "Una institución educativa diseñada para la instrucción, el examen o ambos, de estudiantes en muchas ramas del aprendizaje avanzado, otorgando títulos en diversas facultades y, a menudo, incorporando universidades e instituciones similares". (p. 45). Por lo tanto, se realizó una búsqueda, que se resume en la tabla $\mathrm{N}^{\circ} 2$ de definiciones propuestas por dif erentes autores. (Ver Tabla $\mathrm{N}^{\circ} 2$ ).

Tabla $N^{\circ}$ 2. Definiciones de Universidad Corporativa

\begin{tabular}{|l|l|l|}
\hline Año & \multicolumn{1}{|c|}{ Definición } & \multicolumn{1}{|c|}{ Autor } \\
\hline 1987 & $\begin{array}{l}\text { Una institución cuya misión principal no es la educación, sino la formación } \\
\text { ofrecida por una empresa o industria a sus propios empleados. }\end{array}$ & $\begin{array}{l}\text { Nash } \\
\text { y Hawthorne } \\
(1987)\end{array}$ \\
\hline 1997 & $\begin{array}{l}\text { Una función o departamento que se orienta estratégicamente hacia la } \\
\text { integración del desarrollo de las personas como individuos, con su } \\
\text { desempeño como equipos y finalmente como una organización completa, } \\
\text { mediante la vinculación con los proveedores, mediante la realización de una } \\
\text { amplia investigación, facilitando la entrega de contenido y liderando el } \\
\text { esfuerzo para construir un equipo de alto rendimiento. }\end{array}$ & $\begin{array}{l}\text { Denster (1998) } \\
\text { Meister (1999) }\end{array}$ \\
\hline 1998 & $\begin{array}{l}\text { Es el paraguas estratégico para ofrecer soluciones de aprendizaje para cada } \\
\text { grupo de trabajo en la organización. Una universidad corporativa es } \\
\text { responsable de dar forma a la cultura corporativa y fomentar el desarrollo de }\end{array}$ & 1) \\
\hline
\end{tabular}

\footnotetext{
"Visión de Futuro" Año 16, Volumen N 23 N¹, Enero - Junio 2019 - Pág. 1 - 23

URL de la Revista: http://revistacientifica.fce.unam.edu.ar/

URL del Documento: http://revistacientifica.fce.unam.edu.ar/index.php?option=com content\&view=article\&id=492\&ltemid=101

ISSN 1668 - 8708 - Versión en Línea

E-mail: revistacientifica@fce.unam.edu.ar
} 


\begin{tabular}{|c|c|c|}
\hline Año & Definición & Autor \\
\hline & $\begin{array}{l}\text { habilidades intangibles como liderazgo, pensamiento creativo y resolución de } \\
\text { problemas. }\end{array}$ & \\
\hline 1999 & $\begin{array}{l}\text { Consiste en formar y desarrollar el talento humano en la gestión empresarial, } \\
\text { promover la gestión del conocimiento organizacional (generación, asimilación, } \\
\text { difusión y aplicación) a través de un proceso de aprendizaje activo y continuo. }\end{array}$ & Eboli (1999, p. 25) \\
\hline 1999 & $\begin{array}{l}\text { Proporcionar a la organización un vehículo efectivo para ordenar, facilitar y } \\
\text { nutrir los procesos que apoyan y desarrollan una cultura de aprendizaje dentro } \\
\text { de la organización. Es la encargada de liderar las iniciativas de conocimiento } \\
\text { y aprendizaje de la organización y deberá ser mucho más proactiva e } \\
\text { inclusiva, y estará en el centro de la organización y su toma de decisiones. }\end{array}$ & $\begin{array}{l}\text { Stewart (1999) y } \\
\text { Walton (1999) }\end{array}$ \\
\hline 1999 & $\begin{array}{l}\text { Se pueden ver como un foco para facilitar las prácticas sociales, tecnológicas } \\
\text { y organizacionales que apoyan la creación de conocimiento y el aprendizaje } \\
\text { organizacional, se relaciona con la forma en que las personas tienen sentido } \\
\text { y por lo tanto, aprenden a través de sus experiencias de trabajo. }\end{array}$ & $\begin{array}{l}\text { Schwandt y } \\
\text { Marquardt (1999) }\end{array}$ \\
\hline 1999 & $\begin{array}{l}\text { Una institución educativa diseñada para la instrucción, el examen o ambos, } \\
\text { de estudiantes en muchas ramas del aprendizaje avanzado, otorgando títulos } \\
\text { en diversas facultades y, a menudo, incorporando universidades e } \\
\text { instituciones similares. }\end{array}$ & Lester (1999) \\
\hline 2000 & $\begin{array}{l}\text { Es un proceso orientado a resultados permanentes, que nace a partir de } \\
\text { responder la siguiente pregunta: ¿Qué necesitan saber los trabajadores para } \\
\text { desempeñarse adecuadamente en sus respectivos cargos? }\end{array}$ & Lorenzatti (2000) \\
\hline 2002 & $\begin{array}{l}\text { Desarrolla las habilidades de los empleados y la integra en la orientación } \\
\text { estratégica de la corporación con un fuerte énfasis en liderazgo y ¡Mejora en } \\
\text { el desempeño relacionado con el trabajo! }\end{array}$ & El-Tannir (2002) \\
\hline 2002 & $\begin{array}{l}\text { Está alineado con los conceptos de gestión del conocimiento y aprendizaje } \\
\text { organizacional, y debe ser vista como un foco para la comunicación y } \\
\text { facilitación de prácticas sociales, tecnológicas y organizacionales que apoyan } \\
\text { el aprendizaje y el aprendizaje de la organización. Proceso de creación de } \\
\text { conocimiento, la gestión del talento humano. }\end{array}$ & $\begin{array}{l}\text { Prince y Stewart } \\
(2002)\end{array}$ \\
\hline 2002 & $\begin{array}{l}\text { Son entidades educativas que se constituyen como una herramienta } \\
\text { estratégica diseñada para ayudar a sus organizaciones en la consecución de } \\
\text { sus objetivos, a través de actividades que fomenten el aprendizaje y el } \\
\text { conocimiento a nivel individual y organizacional. }\end{array}$ & Allen, (2002) \\
\hline 2003 & $\begin{array}{l}\text { Son instituciones de aprendizaje, propiedad de la empresa, que apoyan la } \\
\text { gestión estratégica en las empresas mediante programas de desarrollo de } \\
\text { personal, vinculados con el desarrollo estratégico que involucra el aprendizaje } \\
\text { y la acción estratégica y se convierten en un proceso integral. }\end{array}$ & Andresen (2003) \\
\hline 2005 & $\begin{array}{l}\text { La empresa se convierte en una academia, tiene su propio plan de estudios y } \\
\text { capacidad de enseñanza; opera, también investiga. Es irrelevante si la } \\
\text { universidad tiene responsabilidades estratégicas. }\end{array}$ & $\begin{array}{l}\text { Klumpp / } \\
\text { Helmstädter (2005, } \\
\text { p. 4) }\end{array}$ \\
\hline 2005 & $\begin{array}{l}\text { El paraguas organizacional general para alinear y coordinar todo el } \\
\text { aprendizaje para los empleados con el fin de alcanzar los objetivos de la } \\
\text { organización. }\end{array}$ & $\begin{array}{l}\text { Plompen, (2005, p. } \\
83)\end{array}$ \\
\hline 2006 & $\begin{array}{l}\text { Está creada para difundir el conocimiento y desarrollar la competencia } \\
\text { (Coverage), para después llegar a ser un incentivo de despliegue de la } \\
\text { estrategia de empresa (Leverage). }\end{array}$ & Grenzer (2006) \\
\hline 2008 & $\begin{array}{l}\text { Es una institución educativa innovadora con un modelo de negocio, que } \\
\text { integra el desarrollo del personal con el desarrollo de la corporación, el } \\
\text { aprendizaje organizacional y el desarrollo estratégico de la empresa. }\end{array}$ & Sycheva, (2008) \\
\hline 2012 & $\begin{array}{l}\text { Son herramientas para la implementación de la estrategia de la compañía y } \\
\text { su propósito fundamental es la mejora del desempeño, tanto de la empresa } \\
\text { como conjunto y sus áreas de Negocio, como de las personas que colaboran } \\
\text { en la consecución de sus objetivos. }\end{array}$ & Rubio (2012) \\
\hline 2015 & $\begin{array}{l}\text { El propósito de una Universidad Corporativa se puede resumir en tres } \\
\text { ámbitos: Desarrollo de líderes para el futuro; Contribución al desarrollo, } \\
\text { integración y apoyo a la implementación de la estrategia de la organización. }\end{array}$ & Vives et al (2015) \\
\hline 2016 & $\begin{array}{l}\text { Las principales funciones de la Universidad Corporativa son la capacitación } \\
\text { de empleados de diferentes niveles, la gestión del conocimiento, la formación } \\
\text { de valores corporativos comunes, el desarrollo de la cultura corporativa, la } \\
\text { promoción de la innovación. }\end{array}$ & $\begin{array}{l}\text { Lytovchenko (2016 } \\
\text { p. 41) }\end{array}$ \\
\hline 2018 & $\begin{array}{l}\text { Están orientadas al desarrollo de nuevas actividades empresariales y la } \\
\text { creación de nuevas estructuras y relaciones organizacionales. }\end{array}$ & Barrow, (2018) \\
\hline
\end{tabular}

Fuente: Elaboración propia en base a los autores citados (2018)

Del cuadro anterior, encontramos que una característica común clave en las definiciones es la

inclusión de alguna variante de la palabra estrategia; aprendizaje organizacional; mejorar el "Visión de Futuro" Año 16, Volumen No 23 N¹, Enero - Junio 2019 - Pág. 1 - 23

URL de la Revista: http://revistacientifica.fce.unam.edu.ar/

URL del Documento: http://revistacientifica.fce.unam.edu.ar/index.php?option=com content\&view=article\&id=492\&Itemid=101

ISSN 1668 - 8708 - Versión en Línea

E-mail: revistacientifica@fce.unam.edu.ar 
desempeño laboral y la gestión del talento humano. La dificultad central para definir con precisión el término para Meister (1998) proviene del hecho de que una gama diversa de organizaciones usa la nomenclatura de la Universidad Corporativa como una designación general para sus actividades formales de aprendizaje. El término agrega legitimidad y peso, y sugiere una atmósfera de educación y aprendizaje de gestión considerada. El problema se agrava ya que varios de los principales ejemplos del concepto no usan el término para describir sus actividades de aprendizaje, un buen ejemplo es el Banco de Montreal. Una encuesta de Meister (1998) encontró que el 54\% de las llamadas Universidades Corporativas no tenían la palabra universidad en su título. Las designaciones como Instituto de Aprendizaje o Academia de Aprendizaje parecen ser alternativas cada vez más favorecidas.

Esto refleja la naturaleza ambigua del concepto de Universidad Corporativa y se reconoce que tales organizaciones existen en diversas formas, operan bajo una amplia gama de títulos y llevan a cabo un amplio espectro de actividades de educación, capacitación y desarrollo. De hecho, como señala Lester (1999), el término Universidad Corporativa parece ser intercambiable con una variedad de alternativas tales como: Universidad Virtual (Sistemas BAE), Escuela de Negocios Corporativos (Ernst \& Young) y Learning Center (General Motors). Se argumenta, por lo tanto, que lo importante aquí no es el título otorgado a estos organismos, sino el enfoque filosófico de la organización y su percepción de ellos como agentes cruciales para facilitar el aprendizaje y el desarrollo de los empleados.

Tradicionalmente, plantea El-Tannir (2002), que el propósito de una Universidad Corporativa ha estado determinado por la necesidad de capacitar a los empleados y desarrollar sus habilidades generales. Era simplemente una denominación alternativa para los cursos de capacitación organizados de manera básica y distribuir esos programas entre los colaboradores de la organización, en forma de catálogos, de los cuales el personal elegía los cursos y se inscribía en la actividad de capacitación correspondiente. Dichos cursos generalmente se subcontrataban de universidades $u$ otros proveedores de capacitación.

Esta función, sin embargo, se ha encaminado a incorporar una mayor relevancia a las necesidades comerciales inminentes. Se identificaron varias direcciones estratégicas como impulsores del nuevo rol de las universidades corporativas. Andresen e Irmer (1999) por ser:

- Un enfoque impulsado por iniciativa, donde los servicios de UC facilitan una iniciativa corporativa amplia o un proyecto de plan de negocios, tales como iniciativas sobre globalización, productividad y reingeniería de procesos. Andresen an Irmer (1999)

- Un catalizador de gestión de cambios que ayuda a dar forma y completar un proceso transitorio para la empresa, como emprender una nueva estrategia o llevar a cabo una fusión o un proceso de adquisición. Andresen an Irmer (1999)

- Un agente de desarrollo de liderazgo para mantener a los nuevos gerentes con nuevas herramientas para el liderazgo y el fortalecimiento de la gestión interna corporativa.

\footnotetext{
"Visión de Futuro" Año 16, Volumen No 23 N¹, Enero - Junio 2019 - Pág. 1 - 23

URL de la Revista: http://revistacientifica.fce.unam.edu.ar/

URL del Documento: http://revistacientifica.fce.unam.edu.ar/index.php?option=com content\&view=article\&id=492\&Itemid=101

ISSN 1668 - 8708 - Versión en Línea

E-mail: revistacientifica@fce.unam.edu.ar
} 
- Un instrumento de desarrollo de negocios que explora y desarrolla nuevas oportunidades comerciales y motiva a los empleados. Andresen an Irmer (1999)

- Una herramienta de gestión de relaciones cliente-proveedor que se centra en la integración de estándares en la cadena de suministros, así como en la demostración de empleados con habilidades de negociación y gestión de relaciones. Andresen an Irmer (1999)

- Una instalación de desarrollo de carrera basada en la competencia que se enfoca únicamente en los objetivos estratégicos de la corporación y en retener a los empleados a través de estrategias que promueven el aprendizaje permanente. Andresen an Irmer (1999)

Como argumenta Walton (1999), sería justo decir que prácticamente ninguna de las universidades corporativas cumpliría los requisitos establecidos en esta definición, ni tampoco lo desearían. Como Thomas (1999) ha sostenido, en el contexto de la Universidad Corporativa, el término universidad se usa más por sus cualidades simbólicas y de aspiraciones al posicionar el aprendizaje dentro de una organización, que por cualquier intento de imitar las prácticas universitarias tradicionales.

La raíz del fenómeno de la Universidad Corporativa para Taylor y Phillips (2002) es que ven a la Universidad Corporativa de forma diferente a lo que fueron los departamentos de capacitación, ya que forman parte de la capacidad de la organización para cambiarse a sí misma, y reportan directamente al Director Ejecutivo en lugar del Director de Recursos Humanos, por razones de estatus, credibilidad, cultura y enfoque, buscando la educación y el trabajo en conjunto, para el beneficio mutuo de todos. Meister (1998) define una Universidad Corporativa como: el paraguas estratégico para formar y educar a los empleados y sus cuadros con objeto de satisfacer los fines (de la corporación).

Tras su implementación completa, la Universidad Corporativa será el principal vehículo de desarrollo profesional de los empleados de la organización. Facilitará oportunidades de aprendizaje formales e informales que fomentarán el crecimiento personal y profesional de los individuos en la corporación, en un clima organizativo respetuoso, de apoyo y positivo.

El análisis de los trabajos de Fresina (1997), Densford (1998), Meister (1998) y Robie (1999) sugiere que una característica clave de una Universidad Corporativa es el enfoque en el cumplimiento de objetivos y prioridades organizacionales en lugar de los enfoques tradicionales de capacitación y desarrollo, que se basan en la satisfacción de las necesidades individuales. Si se acepta esta proposición, entonces se puede avanzar en una definición mucho más amplia de una Universidad Corporativa moderna, como: una función o departamento que se orienta estratégicamente hacia la integración del desarrollo de las personas como individuos en su desempeño como equipos y finalmente, como una organización completa, mediante la vinculación con los proveedores, a través de la realización de una amplia investigación, facilitando la entrega de contenido y liderando el esfuerzo para construir un equipo de alto rendimiento.

Esta interpretación permite entender a la Universidad Corporativa no como una entidad física, sino como un concepto utilizado para denotar el aprendizaje organizado para el beneficio de la 
empresa. Esto lleva a Meister (1998) y Bachler (1995) a considerar tales instituciones más como facilitadoras de procesos organizacionales, que como entidades organizacionales.

Basándose en el modelo OLS de Schwandt y Marquardt (1999), las universidades corporativas se pueden ver como un medio para facilitar las prácticas sociales, tecnológicas y organizacionales que apoyan la creación de conocimiento y el aprendizaje organizacional. Se argumenta que la idea de una universidad corporativa se relaciona con la forma en que las personas tienen sentido en y para la organización y por lo tanto, aprenden a través de sus experiencias de trabajo.

De esta manera una Universidad Corporativa, podría proporcionar a la organización un vehículo efectivo para ordenar, facilitar y nutrir los procesos que apoyan y desarrollan una cultura de aprendizaje dentro de la organización. Es la encargada de liderar las iniciativas de conocimiento y aprendizaje de la organización, deberá ser mucho más proactiva e inclusiva, y estará en el centro de la organización y su toma de decisiones; como sostienen Stewart (1999) y Walton (1999), la incapacidad de las empresas para ofrecer aprendizaje organizacional en un sentido significativo se debe en parte a su incapacidad para integrar y coordinar una amplia gama de funciones, actividades y procesos.

En una conceptualización alternativa de la Universidad Corporativa, Prince y Stewart (2002) se centran en los procesos más que en los resultados y la estructura. Poniendo atención al contexto, incorporan cuatro subprocesos de aprendizaje de las organizaciones, que están coordinados e integrados por la Universidad corporativa para facilitar el aprendizaje organizacional. Estos subprocesos son: sistemas de conocimiento y procesos, redes y asociaciones, procesos de aprendizaje y procesos de personas. Aquí la atención se centra en proporcionar "un dispositivo descriptivo y analítico" (Prince y Stewart, 2002, p. 794) en lugar de un tipo ideal. Los autores citados proponen que el futuro de las universidades corporativas depende de su capacidad para gestionar la interacción y la complejidad de los subsistemas de aprendizaje. Si bien el e-learning no se menciona específicamente, es posible que pueda contribuir a uno de estos subprocesos de aprendizaje, sino a todos.

Toda empresa es un producto de la historia y las circunstancias de la creación de la Universidad Corporativa cambian independientemente. Según Grenzer, J. (2006) se puede destacar como condición sine que non la voluntad expresada desde el nivel más alto; esta voluntad resuelta de una intuición, de una visión del futuro y de los desafíos que el futuro reserva para la empresa.

Por otra parte, la definición de Universidad Corporativa (UC) propuesta por El-Tannir (2002) es: una función o departamento en la compañía que desarrolla las habilidades de los empleados y las integra en la orientación estratégica de la organización con un fuerte énfasis en liderazgo y ¡Mejora en el desempeño relacionado con el trabajo! Las perspectivas de las UC se aclaran cada vez más como la elección correcta para el desarrollo continuo de los empleados hacia una formación más específica que mejore el rendimiento del personal y aumente la productividad en sus trabajos.

\footnotetext{
"Visión de Futuro" Año 16, Volumen No 23 N¹, Enero - Junio 2019 - Pág. 1 - 23

URL de la Revista: http://revistacientifica.fce.unam.edu.ar/

URL del Documento: http://revistacientifica.fce.unam.edu.ar/index.php?option=com content\&view=article\&id=492\&Itemid=101

ISSN 1668 - 8708 - Versión en Línea

E-mail: revistacientifica@fce.unam.edu.ar
} 
Para Steck (2003) la Universidad Corporativa se concibe como una institución que se caracteriza por procesos, criterios decisionales, expectativas, cultura organizacional y prácticas operativas que se toman de, y tienen sus orígenes en, la corporación comercial moderna.

El centro de la Universidad Corporativa será con base en Dealtry (2005), en gran parte la propiedad y estará basada en una sólida red de personas ubicadas en diferentes actividades o funciones en toda la organización; todos tendrán la oportunidad de lograr habilidades efectivas en aprender a aprender en línea dentro de la realidad de los eventos de sus departamentos o actividades; habrá un cuadro voluntario de personas que proporcionará la infraestructura de apoyo para los estudiantes (por ejemplo, clientes del proyecto, mentores, entrenadores, compañeros de estudio, etc.).

Para Dealtry (2005) estará soportada en una solución de e-learning distribuida y eficiente, con contenidos de calidad, habrá una infraestructura basada en nuevas tecnologías de la información y la comunicación accesible para todos, con instalaciones para formar y llevar a cabo comunidades de aprendizaje y de mejores prácticas en torno a las principales disciplinas.

Como complemento Dealtry (2005) establece que se dispondrá de una base de datos de búsqueda de conocimientos para las lecciones aprendidas, en base a las comunidades de mejores negocios y prácticas administrativas; las actividades de las universidades corporativas se convertirán en parte integrante del plan de desarrollo profesional de cada individuo y serán revisadas como un elemento clave dentro del trabajo y la evaluación del desempeño; habrá sistemas de Acreditación del Aprendizaje Experiencial Previo (Accreditation of Prior Experiential Learning , APEL ${ }^{1}$ ) y Apreciación de la trayectoria profesional (Career Path Appreciation, $\mathrm{CPA}^{2}$ ) a través de los cuales propone Dealtry (2005) que cada persona puede identificar y planificar su aprendizaje basado en el trabajo; tanto el aprendizaje formal como el informal serán recompensados con créditos profesionales que contarán en las revisiones salariales y de promoción.

Por su parte Grenzer (2006) pone de relieve algunos puntos que son requisitos al momento de crear una Universidad Corporativa. Se habla del desarrollo de la prestación a nivel del individuo, para migrar progresivamente hacia un nivel colectivo y después organizacional. Además, la Universidad Corporativa está creada para difundir el conocimiento y desarrollar la competencia (Coverage) a fin de llegar a ser un incentivo de despliegue de la estrategia de empresa (Leverage). En fin, si la universidad queda centrada sobre su misión de desarrollo de las competencias individuales tales que, prescritas notamente por el entorno legislativo, se encuentra prisionera del cuadrante formación y no extiende su territorio de intervención al servicio de la organización.

$1 \quad$ Es el reconocimiento formal (basado en la evaluación profesional) del aprendizaje adquirido de la experiencia previa, por lo general de la experiencia no relacionada con un contexto académico.

2 El CPA (Apreciación de la trayectoria profesional), basado en la matriz de relaciones de trabajo (Matrix of Working Relationships MWR), es una metodología de entrevista diseñada para identificar la capacidad de un individuo para trabajar en niveles predeterminados de complejidad, tanto en el momento de la entrevista como en momentos específicos en el futuro.

"Visión de Futuro" Año 16, Volumen N²3 N¹, Enero - Junio 2019 - Pág. 1 - 23

URL de la Revista: http://revistacientifica.fce.unam.edu.ar/

URL del Documento: http://revistacientifica.fce.unam.edu.ar/index.php?option=com content\&view=article\&id=492\&Itemid=101

ISSN 1668 - 8708 - Versión en Línea

E-mail: revistacientifica@fce.unam.edu.ar 
Tal como explica Alfaro, (2012) antes de pensar en crear una Universidad Corporativa, se ha dado cuenta de varios puntos: era una organización muy técnica, con un gran conocimiento no compartido, era una organización dispersa al nivel geográfico, una mala estructura de la organización desde el punto de vista de la gestión del conocimiento, etc.; con el objetivo principal de encontrar un proyecto estratégico y sostenible.

Para Barrow, (2018) las Universidades Corporativas están orientadas al desarrollo de nuevas actividades empresariales, a la creación de nuevas estructuras y relaciones organizacionales. Un elemento central de su argumento es la premisa de que la burocracia corporativa resultante de estas actividades está en tensión con la actividad empresarial y amenazan la estructura de poder y la lógica de la Universidad Corporativa.

A partir de estos resultados, debe quedar claro que las actividades de la Universidad Corporativa no están destinadas a socavar en modo alguno las funciones y actividades principales de las organizaciones, sino que son completamente complementarios. Son esencialmente facilitadoras e integrales y traen formalmente las capacidades de aprendizaje en tiempo real y economía del conocimiento transferibles, agregando profundidad y calidad a la experiencia laboral de un individuo en la vanguardia del negocio.

Finalmente, en este sentido, y tomando como base a Meister (1998), Lorenzatti (2000); ElTannir (2002); Prince y Stewart (2002); Allen, (2002); Prince y Stewart (2002); Andresen (2003); Rubio (2012); Lytovchenko (2016); Barrow, (2018); el concepto de Universidad Corporativa que utilizaremos en esta investigación está alineado con el desarrollo de la estrategia de la organización y con los conceptos de gestión del conocimiento, que deben ser vistos como un medio para la comunicación y facilitación de prácticas sociales, tecnológicas y organizacionales que apoyan el aprendizaje individual y el aprendizaje de la organización, mediante la gestión del talento humano y procesos de creación de conocimiento. Su éxito depende del compromiso del líder y su capacidad para gestionar y aprovechar la compleja interacción de los subsistemas de aprendizaje organizacional, orientados a la mejora del desempeño laboral y encarnan la identidad, la cultura y la marca de la organización para todos sus grupos de interés.

\footnotetext{
"Visión de Futuro" Año 16, Volumen No 23 N¹, Enero - Junio 2019 - Pág. 1 - 23

URL de la Revista: http://revistacientifica.fce.unam.edu.ar/

URL del Documento: http://revistacientifica.fce.unam.edu.ar/index.php?option=com content\&view=article\&id=492\&Itemid=101

ISSN 1668 - 8708 - Versión en Línea

E-mail: revistacientifica@fce.unam.edu.ar
} 


\section{Modelo de Universidad Corporativa}

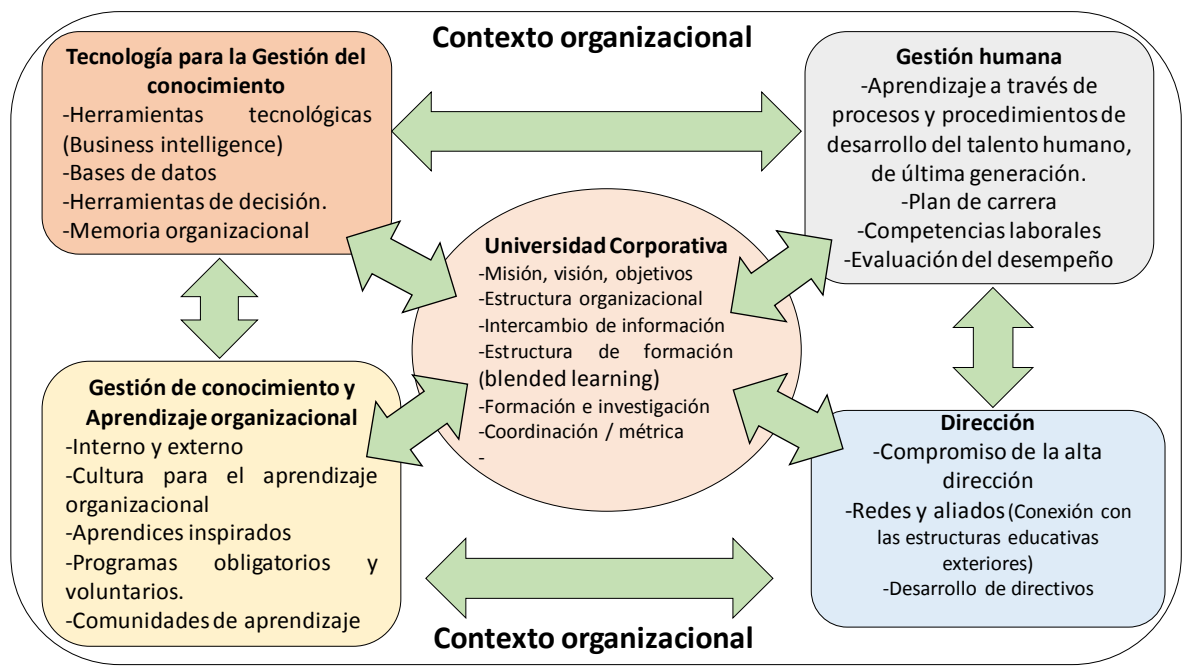

Figura $\mathrm{N}^{\circ}$ 2. Modelo de Universidad Corporativa

Fuente: Elaborado por Garzón (2018) en base a Prince y Stewart, 2002; Blackburn, C., y Tétreault, S. (2013); Equilbey, N. y Boyer, L. (2013); Cristol, y Mellet, (2013); Meier, O. (2012); Vincent. (2012); Labruffe, A. (2012); Abel, y Li, (2012) Soparnot,(2012); Cadin, (2012); Falek, H. (2010); Rothwell, W. (2010); Soparnot, R. (2010); Beaujolin,(2010.); Hosdey, A. y Rogister, J.( 2009; Barmeyer y Waxin (2008); Wheeler, K. (2005); Shaw, (2005).;Walton (2005); Allen, M. (2002); Saussereau y Stepler (2002); Jarvis, P. (2001).;Durand, C., Fili, y Hénault, (2000); Finn, W. (1999); Finn, (1999); Fresina (1997); Barley, K. (1997)

La Figura N2 del modelo de Universidad Corporativa, muestra la relación entre cuatro procesos clave de la Universidad Corporativa: la dirección y el compromiso de la alta dirección; la gestión del conocimiento y el aprendizaje organizacional; la gestión humana; y las tecnologías para la gestión del conocimiento. Proporciona una buena descripción de los medios de intercambio de aprendizaje que existen entre los procesos centrales y que ayudan a explicar las acciones en términos de sus contribuciones al aprendizaje organizacional.

La evidencia sugiere que, si bien muchas de las universidades corporativas de hoy en día pueden no llevar a cabo todas estas funciones, la necesidad de desarrollar organizaciones creadoras de conocimiento conduce a universidades corporativas más ambiciosas que probablemente estén más involucradas en la toma de decisiones operativas y estratégicas de las organizaciones. Su éxito dependerá de su capacidad para gestionar y aprovechar la compleja interacción de los subsistemas de aprendizaje organizacional y menos de su capacidad para administrar programas de capacitación y educación.

\section{La Dirección}

En la medida en que la Universidad Corporativa da respuesta a su finalidad, será decisiva para el futuro de la empresa la implicación activa de los directivos. Según Blackburn, C., y Tétreault, S. (2013), la implicación de los directivos de la empresa es fundamental y los miembros del comité de dirección requieren la definición de las prioridades y de los temas potenciales de programas de prácticas.

\footnotetext{
"Visión de Futuro" Año 16, Volumen No 23 N¹, Enero - Junio 2019 - Pág. 1 - 23

URL de la Revista: http://revistacientifica.fce.unam.edu.ar/

URL del Documento: http://revistacientifica.fce.unam.edu.ar/index.php?option=com content\&view=article\&id=492\&Itemid=101

ISSN 1668 - 8708 - Versión en Línea

E-mail: revistacientifica@fce.unam.edu.ar
} 
Los programas de las universidades corporativas, para Falek, H. (2010), deben definirse con la participación activa de los directivos y de los responsables de las principales áreas funcionales de la empresa. Más allá de la concepción, de la organización y de la motivación a participar en los programas previstos, el funcionamiento de la Universidad Corporativa implica otros aspectos. Primero, según Rothwell, W. (2010), una coordinación estrecha con la gestión del talento humano (reclutamientos, selección, contratación, desarrollo y promociones) para lo cual es fundamental el paso por la UC, que constituye una etapa obligada del desarrollo de la carrera. También, requiere una coordinación estrecha con los responsables operacionales y el seguimiento, en el terreno de la acción de formación, es importante. Es significativo también hacer una búsqueda y una reflexión prospectiva sobre la evolución de los puestos de trabajo y sobre los medios susceptibles de mejorar la calidad de los productos y servicios que constituyen la razón de ser de la empresa.

Por lo tanto, el compromiso de la alta dirección, para Barmeyer y Waxin, (2008) requiere que sea participante o animador, lo que depende de la actitud del directivo, como sucedió en General Electric, con Jack Welch (antiguo presidente del grupo) quien dio un ejemplo de la implicación del CEO (chief executive officer) en la Universidad Corporativa GE, quine intervenía regularmente, observando con atención a los participantes, lo que le permitía a la vez asegurarse de la difusión de la estrategia y de localizar los talentos prometedores.

Como resultado de esta implicación del presidente de la organización, Barmeyer y Waxin, (2008) afirman que GE ha formado más altos directivos de empresas que algunas de las Escuelas de Negocios de mayor reputación. Los altos dirigentes también pueden pasar de manera informal para sentir la moral de los grupos y asegurarse de que los mensajes de la dirección estén correctamente recibidos y aplicados, logrando poner de relieve que no es fácil para los responsables de la Universidad Corporativa encontrar el buen equilibrio entre las intervenciones de los expertos externos y las de los dirigentes.

\section{Gestión del conocimiento y aprendizaje organizacional}

Las universidades corporativas se conectan con la gestión del conocimiento y el aprendizaje organizacional, e impactan en los procesos de creación de conocimiento como un componente necesario de esa función, esto es un imperativo para la noción de universidades corporativas, lo cual se puede evidenciar en Starbuck (1992) quien propone un crecimiento de lo que se denomina empresas de conocimiento intensivo y Hamel y Prahalad (1990), Drucker (1993) y Blackler (1993) argumentan que el conocimiento es una fuente importante de ventaja competitiva, por lo tanto, ese conocimiento es central para la creación de riqueza, que se describen a sí mismas como en el negocio del conocimiento, que van desde Britis Petroleum Co (BP), que perfora en busca de petróleo hasta Senco, que fabrica clavos.

Estos desarrollos en la naturaleza de la producción tienen implicaciones e impacto en las estructuras organizacionales y el diseño del trabajo en las organizaciones, de acuerdo a Prusak, (1997); Stewart, (1997); Coulson-Thomas, (1997); Davenport y Prusak, (1998). Scarborough et al. 
(1999) señalan que estos cambios crean nuevos problemas para el aprendizaje y el desarrollo de conocimientos especializados, incluidas las oportunidades para el intercambio casual de conocimientos.

Por lo tanto, se puede argumentar sensatamente que una tarea clave para las universidades corporativas es proporcionar un vehículo para construir un significado compartido a través de influir y controlar los procesos de aprendizaje y la creación de conocimiento. El último de estos puede caracterizarse como el dominio de la gestión del conocimiento. Si bien Bell (1973) ya señaló por primera vez la importancia del conocimiento en la era postindustrial, como una competencia crítica para la gestión de las organizaciones (Ruggles, (1998); Walton, (1999)).

Con relación a la gestión del conocimiento, Garzón (2006) y Scarborough et al. (1999) la definen como: cualquier proceso o práctica de crear, adquirir, capturar, compartir y usar el conocimiento, donde sea que se encuentre, para mejorar el aprendizaje y el desempeño en las organizaciones. Esta es una definición más bien abarcativa, que hace difícil imaginar lo que podría no ser la gestión del conocimiento. Este punto de vista es reconocido en cierta medida por Skyrme y Amidon (1997), quienes señalan que la mayoría de las organizaciones se darán cuenta de que han estado administrando el conocimiento para el bien corporativo durante muchos años. Sin embargo, continúan argumentando que hay tres nuevos desarrollos: (1) hacer que los procesos de conocimiento y el conocimiento sean más explícitos; (2) el desarrollo de marcos estratégicos para guiar la explotación del conocimiento; y (3) la introducción de métodos más sistemáticos para la gestión del conocimiento (Skyrme y Amidon, 1997).

En el modelo de Earle (1994), esta dimensión representa el intento de la organización de crear una organización de aprendizaje basada en la creación de una cultura de aprendizaje respaldada por la educación y la capacitación. Hasta ahora, se ha establecido que los conceptos significativos de gestión del conocimiento, aprendizaje organizacional y organización del aprendizaje son complejos y multifacéticos, por lo tanto, las universidades corporativas se pueden ver como un foco para facilitar las prácticas sociales, tecnológicas y organizacionales que apoyan la creación de conocimiento y el aprendizaje organizacional y se relaciona con la forma en que las personas tienen sentido y significado, por ende, aprenden a través de sus experiencias de trabajo.

\section{Gestión humana}

La Universidades Corporativas, se conciben como una herramienta para ayudar a desarrollar el talento humano, preparándolos para los retos y oportunidades a los que sus organizaciones se tendrán que enfrentar en el futuro, de esta manera, la gestión del talento busca el alineamiento de los recursos humanos con el futuro de las organizaciones, para lo cual debe disponer de las adecuadas bases de información necesarias para la gestión de su actividad con indicadores de gestión integrados en las bases de datos del Negocio y suponen una apuesta firme por el capital humano y sus competencias como ventaja competitiva.

\footnotetext{
"Visión de Futuro" Año 16, Volumen No 23 N¹, Enero - Junio 2019 - Pág. 1 - 23

URL de la Revista: http://revistacientifica.fce.unam.edu.ar/

URL del Documento: http://revistacientifica.fce.unam.edu.ar/index.php?option=com content\&view=article\&id=492\&Itemid=101

ISSN 1668 - 8708 - Versión en Línea

E-mail: revistacientifica@fce.unam.edu.ar
} 
En este contexto, surgen las universidades corporativas como respuesta a las necesidades concretas de formación empresarial, no solo de capacitación técnica sino también gerencial, a la medida de cada negocio y apuntando de manera clara a sus estrategias (Jarvis, 2006). De manera general, para encontrar el éxito, la Universidad Corporativa debe estar considerada como una inversión a largo plazo y quedar posicionada a nivel estratégico, que generalmente es responsabilidad del talento humano, aunque existen cada vez más casos en los cuales las universidades corporativas le reportan directamente al presidente de la organización. De esta manera los departamentos de Gestión Humana, son un socio legítimo de la universidad corporativa y deben servir a las prioridades estratégicas de la empresa.

Para Cadin, L. (2012) es responsabilidad de la Gestión Humana vigilar el compromiso, la atracción de la empresa en general y los programas en particular, el reclutamiento y el compromiso de los potenciales talentos, así como la contribución de la universidad en la gestión de los planes de sucesión. Con las herramientas colaborativas, los recursos humanos están también cada vez más atentos al comparto de las buenas prácticas, a la emergencia y a la animación de las redes internas para que la función de la universidad corporativa debe aprehender claramente el conjunto de las dimensiones que constituyen su misión: formación de adultos, promoción de valores y de la cultura de empresa.

Para Abel, y Li, (2012) las Universidades Corporativas (UC) existen en todo tipo de organizaciones y en todos los tamaños y formas. Al mismo tiempo, la mayoría de las UC siguen siendo iniciativas impulsadas por las empresas. Cuanto más dependa una organización de la competitividad de su talento humano para competir en el mercado global, más énfasis debe poner en el desarrollo de sus colaboradores.

Finalmente, bajo esta perspectiva, según Allen, (2002) la gestión humana debe sustentar su labor formativa en 3 funciones esenciales: la función académica como fundamento de la formación del capital humano, la función de investigación y desarrollo como soporte de formación del capital intelectual y la función de extensión y difusión sustentada en alianzas positivas y responsabilidad social. Son estos tres ejes que soportan el trabajo de evaluación presentado en este documento.

\section{Tecnologías para la gestión de conocimiento en las universidades corporativas}

Las universidades corporativas se enriquecen según Kearsley, (2010), con el uso de diversas modalidades de aprendizaje: presencial, a distancia o virtual y mixta. De la misma manera, contempla el uso de la tecnología de la información y la comunicación (TIC) para apoyar el proceso de formación a través del uso de plataformas electrónicas de aprendizaje (LMS o learning management systems), de interacción y comunicación a distancia y la construcción de repositorios electrónicos de objetos de aprendizaje. Esto con la finalidad de apoyar y reforzar los planes individuales de desarrollo, potencializando de acuerdo a los diferentes estilos de aprendizaje, las diversas estrategias y recursos de formación.

\footnotetext{
"Visión de Futuro" Año 16, Volumen N²3 N¹, Enero - Junio 2019 - Pág. 1 - 23

URL de la Revista: http://revistacientifica.fce.unam.edu.ar/

URL del Documento: http://revistacientifica.fce.unam.edu.ar/index.php?option=com content\&view=article\&id=492\&Itemid=101

ISSN 1668 - 8708 - Versión en Línea

E-mail: revistacientifica@fce.unam.edu.ar
} 
En este sentido, es importante resaltar que para Vives et al. (2015) se están aplicando modelos híbridos (que combinan formación presencial con formación online), haciendo mucho énfasis en la formación en el puesto de trabajo (que busca la aplicación de los conceptos y prácticas entendidos), así como una formación mucho más experiencial de aprender haciendo, o, según su expresión en inglés, learning-by-doing.

De la misma forma Deloite (2016) establece la adopción de nuevas tecnologías y nuevos modelos de aprendizaje como los MOOCs (Massive Open Online Course) cursos en línea abiertos, como una oportunidad de aprendizaje y se convierte en uno de los principales impulsores del compromiso de los colaboradores, y generan un lugar de trabajo con una cultura sólida.

Finalmente es importante considerar que el poder disruptivo y transformador de los MOOC se encuentra en la organización de actividades de aprendizaje que están abiertas a la participación masiva de los estudiantes matriculados en estos cursos. La educación a distancia también podría apoyar la enseñanza presencial. Implica acceso libre, colaboración, reutilización, remezcla, redistribución, inclusión y adaptación (Cabero et al., 2014). Sin embargo, este no es un proceso sencillo. Se necesita más investigación científica para contrarrestar las críticas y superar sus limitaciones (Liyanagunawardena et al., 2013). Además, es necesario analizar los beneficios de las prácticas educativas utilizando MOOC / SPOC para crear espacios que sean más interesantes de aprender. En estos espacios, se podría fomentar la innovación real cambiando la forma en que interactúan los alumnos y el profesorado (Chiappe-Laverde et al., 2015).

\section{CONCLUSIÓN}

En una sociedad globalizada que se encuentra siempre en constante evolución, el dirigente de la Universidad Corporativa debe tomar medidas para hacer frente de manera eficaz a los desafíos que ocurren.

Las universidades corporativas deben hacer frente a nuevas misiones; tales como la elaboración de programas con los clientes internos, acompañamiento individual y de equipo, consejo y coaching de proyectos y acompañamiento del cambio, comoo también la gestión del proceso de innovación con los clientes.

Para el futuro, la Universidad Corporativa primero debe estar orientada a la función de la organización que sirve, debe ofrecer espacios de expresión libres creando las disposiciones de confianza necesarias para el aprendizaje colectivo, que consiste en ponerse a disposición de los directivos para hacer que se encuentren a sí mismos y así crear un efecto de red en la organización. Esta dinámica de aprendizaje puede permitir reducir la distancia entre los dirigentes y sus colaboradores y así mejorar las relaciones y la comunicación organizacional.

De la misma forma, la Universidad Corporativa debe cultivar relaciones de apertura hacia el mundo exterior para estar al día de las evoluciones. Debe enfocarse en el conocimiento y la comprensión de las necesidades de las funciones, también para crear mejor la articulación entre 
aprendizaje e innovación, por lo tanto, deben preparar las funciones y el saber-hacer del futuro, crear las condiciones y dar los medios necesarios a sus colaboradores para innovar.

Por otra parte, la Universidad Corporativa debe adaptar su acción a las necesidades de las nuevas generaciones. En efecto, la universidad debe evolucionar en sus propias prácticas para corresponder mejor a las expectativas de nuevos públicos. Debe desarrollar nuevas formas de aprendizaje y hacer evolucionar el papel del formador en consecuencia. Para abordar estas nuevas generaciones, la universidad necesita capitalizar los conocimientos y el saber-hacer de ayer y de hoy para transmitir un aprendizaje de la cultura de la empresa, de su historia y del enfoque de su función. También, en su rol de detectar los talentos para crear nuevas comunidades de líderes.

Finalmente, la propuesta del modelo de Universidad Corporativa muestra la relación entre cuatro procesos de la Universidad Corporativa clave, la dirección y el compromiso de la alta dirección; la gestión del conocimiento y el aprendizaje organizacional; la gestión humana; y las tecnologías para la gestión del conocimiento, que proporcionan una buena descripción de los medios de intercambio de aprendizaje que existen entre los procesos centrales y que ayudan a explicar las acciones en términos de sus contribuciones al aprendizaje organizacional.

\section{REFERENCIAS}

Abel, L.; Li, J. (2012). Exploring the Corporate University Phenomenon: Development and Implementation of a Comprehensive Survey. Human resource development quarterly. Vol. 23, No1, Spring 2012 @ Wiley Periodicals, Inc. DOI: 10. 1002/hrdq.211

Aguirre, C.; Alvarez-Montefusco, C; Picerno, N. (2017). Las prácticas de la Universidad Corporativa, alineadas a la cultura organizacional. Trabajo de grado de pregrado de la Universidad Argentina de la Empresa.

Allen, M. (2002). The corporate university handbook: Designing, managing, and growing a successful program. New York, NY: AMACOM.

Anderson, L. (2000). Business Education Survey-Corporate universities. Financial Times, 23 de October.

Anderson, L. (2003, March 24). Collaboration, not rivalry, is best way ahead-corporate universities v. business schools. Financial Times.

Andresen, M. (2003). Corporate Universities also Instrument des Strategies change Managements von Person, Gruppe und Organization. Frankfurt, 2003.

Andresen, M. and Irmer A. (1999). Corporate universities in Germany- first experiences, The new corporate University Review, november/december.

Andreu R. (2001). Caso Unión FENOSA, Cambio cultural y aprendizaje en las organizaciones, IESE, Barcelona, España.

Arkin, A. (2000). "Combined honours", People Management, October, pp. 42-4.

\footnotetext{
"Visión de Futuro" Año 16, Volumen No 23 N¹, Enero - Junio 2019 - Pág. 1 - 23

URL de la Revista: http://revistacientifica.fce.unam.edu.ar/

URL del Documento: http://revistacientifica.fce.unam.edu.ar/index.php?option=com content\&view=article\&id=492\&Itemid=101

ISSN 1668 - 8708 - Versión en Línea

E-mail: revistacientifica@fce.unam.edu.ar
} 
Arnone, M. (1998). Corporate universities: a viewpoint on the challenges and best practices, Career Development International, Vol. 3 No. 5, pp. 199-205.

Baldwin, T., Danielson, C., \& Wiggenhorn, W. (1997). The evolution of learning strategies in organizations: From employee development to business redefinition. Academy of Management Executive, 11(4), 47-58.

Barmeyer, C. y Waxin, M. (2008). Gestion des Ressources Humaines Internationales- Problématique, Stratégies et Pratiques. Editions Liaisons. Beaujolin, F. (2001) Vers une organisation apprenante. Editions Liaisons.

Barrow C. (2018). The Entrepreneurial Intellectual in the Corporate University, Publisher Palgrave MacMillan ISBN 978-3-319-63052-6.

Bell, D. (1973). The Coming of Post-Industrial Society, Penguin, Penguin, Harmondsworth.

Blackburn, C. y Tétreault, S. (2013). Le dirigeant et son équipe de managers - Managez mieux, Stressez moins. Gereso Edition Boyer, L. y Equilbey, N. (2013) Evolution des organisations et du management : Rétrospective et prospective. EMS.

Blackler, F. (1993). "Knowledge and the theory of organizations -organizations as activity systems and there-framing of management", Journal of Management Studies, Vol.30 No.6, pp. 863-84.

Bober, C., \& Bartlett, K. (2004). The utilization of training program evaluation in corporate universities. Human Resource Development Quarterly, 15(4), 363-388.

Cabero, J., Llorente, M. D. C., \& Vázquez, A. I. (2014). Las tipologías de MOOC: Su diseño e implicaciones educativas. PRO, 18(1), 13-26 Retrieved from http://hdl.handle.net/10481/31663. Cadin, L. (2012). Gestión des ressources humaines. Dunod.

Chiappe-Laverde, A., Hine, N., \& Martínez-Silva, J. (2015). Literature and practice: A critical review of MOOCs. Media Education Research Journal, 44(22), 9-17. https://doi.org/10.3916/C442015-01.

Coulson-Thomas, C.J. (1997). "The future of the organization", Journal of Knowledge Management, Vol. 1 No. 1, pp. 15-26.

Davenport, T.H. and Prusak, L. (1998). Working Knowledge, Harvard Business School Press, Boston, MA.

Dawson, P., Preece, D. and McLoughlin, I. (2003). "From Essex to cyberspace: virtual organizational reality and real organization virtuality", Labour and Industry, Vol. 14 No. 1, pp. 73-89.

Dealtry, R. (2005). Achieving integrated performance management with the corporate university, Journal of Workplace Learning, Vol. 17 Iss 1/2 pp. 65 - 78 Permanent link to this document: http://dx.doi.org/10.1108/13665620510574469.

Densford, L.E. (1998). Many Corporate Universities under Development: The Aim is to link Training to Business The Corporate University Review, Nov-Dec.

Drucker, P.F. (1993). Post-Capitalist Society, HarperCollins, London.

Earle, M.J. (1994), "Knowledge as strategy: reflections on Skandia International and Shorko Films", in Ciborra, C. and Jelassi, T. (Eds), Strategic Information Systems, John Wiley \& Sons, London.

\footnotetext{
"Visión de Futuro" Año 16, Volumen No 23 N¹, Enero - Junio 2019 - Pág. 1 - 23

URL de la Revista: http://revistacientifica.fce.unam.edu.ar/

URL del Documento: http://revistacientifica.fce.unam.edu.ar/index.php?option=com content\&view=article\&id=492\&Itemid=101

ISSN 1668 - 8708 - Versión en Línea

E-mail: revistacientifica@fce.unam.edu.ar
} 
Eboli, M. (2004). Universidades setoriais unem empresas. Disponível em: http://www.fia.com.br/admpauta/152b/univ_setoriais.htm. Acesso em: 25 de setembro de 2017.

El-Tannir A. (2002)- The corporate university mode for continuous learning, training and development, in Education and training, March 2002, volume 44, number 2, pp.76-81, DOI $10.1108 / 00400910210419973$.

Eurich, N. (1985). Corporate Classrooms: The Learning Business .A Carnegie Foundation Special Report, Jossey-Bass; 1 edition (June 15, 1985).

Falek, H. (2010) Le guide de la formation professionnelle en entreprise. Gualino Editeur.

Fresina, A. (1997)- The Three Prototypes of Corporate Universities- The Corporate University Review, Jan/Feb.

Garbellini L. (2013). Universidades Corporativas: funcionamiento dentro de la empresa, formación de los managers, dimensión internacional, y el impacto sobre el cambio organizacional, Facultad de ciencias económicas y empresariales, Universidad Pontifica de Comillas, Madrid.

Garzón M. (2006). Aproximaciones a la gestión del conocimiento en empresas colombianas, Revista Universidad \&. Empresa, Bogotá (Colombia) 5 (10): 232-256, junio de 2006.

Gómez L. (2018). Universidades Corporativas UC, Avanza.

Grenzer, J. W. (2006). Developing and implementing a Corporate University. HRD Press.

Hamel, G. and Prahalad, C.K. (1990). "The core competence of the corporation", Harvard Business Review, Vol. 68 No. 3, pp. 77-91.

Holland, P., \& Pyman, A. (2006). Corporate universities: A catalyst for strategic human resource development? Journal of European Industrial Training, 30(1), 19-31.

Jansink, F., Kwakman, K., \& Streumer, J. (2005). The knowledge-productive corporate university. Journal of European Industrial Training, 29(1), 40-57.

Jarvis, P. (2006). Universidades corporativas. Nuevos modelos de aprendizaje en la sociedad global. Madrid, España: Editorial Narcea S.A. De ediciones.

Kearsley, G. (2010). Andragogy (M.Knowles). The theory into practice database. Retrieved from http://tip.psychology.org

Klumpp, M. y Helmstädter H. (2005). Campus Sapiens Paper No. 3 Corporate University Definitionen und Konzepte. Essen 2005.

Lester, T. (1999). Degree Culture" Human Resources, March 74-78.

Lipt, D. (2013). Disney U: How Disney University develops the world's most engaged, loyal, and customer-centric employees. New York: McGraw-Hill.

Liyanagunawardena, T. R., Adams, A. A., and Williams, S. A. (2013). MOOCs: A systematic study of the published literature 2008-2012. The International Review of Research in Open and Distributed Learning from http://www.irrodl.org/index.php/irrodl/article/view/1455/2531

\footnotetext{
"Visión de Futuro" Año 16, Volumen No 23 N¹, Enero - Junio 2019 - Pág. 1 - 23

URL de la Revista: http://revistacientifica.fce.unam.edu.ar/

URL del Documento: http://revistacientifica.fce.unam.edu.ar/index.php?option=com content\&view=article\&id=492\&Itemid=101

ISSN 1668 - 8708 - Versión en Línea

E-mail: revistacientifica@fce.unam.edu.ar
} 
Lorenzatti, M. (2000) La Universidad Corporativa llegó para quedarse. Disponible en: http://www.americalearningmedia.com/edicion-006/72-analisis/320-launiversidad-corporativallego-para-quedarse [Consultado el 12 de mayo de 2018, Hora: 3:00pm].

Lytovchenko I. (2016). Corporate university as a form of employee training and development in American companies, Advanced Education, 2016, Issue 5, 35-41 DOI: 10.20535/24108286.6228.

Macpherson, A., Homan, G., \& Wilkinson, K. (2005). The implementation and use of e-learning in the corporate university. Journal of Workplace Learning, 17(1/2), 33-4.

Meister, J. (1997). Market audits of corporate universities, Corporate University Review, Vol. 5 No. 1, pp. 26-34.

Meister, J. (1998). Corporate universities: Lessons in building a world-class work force (2nd ed.). New York, NY: McGraw-Hill.

Nash, Nancy S., \& Hawthorne, Elizabeth M. (1987). Formal Recognition of Employee Sponsored Instruction: conflict and collegiality in postsecondary education. Washington, D.C.: Association for the Study of Higher Education, 1-3.

Peak, M. (1997). Go corporate Universities, Management Review (USA), Vol. 86No.2, pp.33-8.

Phillips, J. (1999). Worldwide Solutions to Competition in a Global Economy, Gulf Publishing Company, Houston, TX.

Plompen, M. (2005). Innovative corporate learning: excellent management development practice in Europe. Houndmills, Basingstoke, Hampshire; New York: Palgrave Macmillan. http://dx.doi.org/10.1057/9780230288799.

Prince, Ch y Stewart, J. (2002). Corporate universities an analytical framework, Journal of

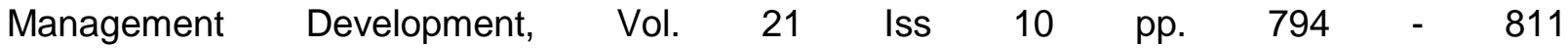
http://dx.doi.org/10.1108/02621710210448057.

Prusak, L. (Ed.) (1997). Knowledge in Organizations, Butterworth-Heinemann, New York, NY.

Rademakers, M. (2005). Corporate universities: Driving force of knowledge innovation. Journal of Workplace Learning, 17(1/2), 130-136.

Rees, G.; y Smith P. (2014). Strategic human resource management, Sage Publications Ltd, London. Ricardo, J. (2007). Gestão da educação corporativa. 1.ed. São Paulo: Pearson Prentice Hall, 2007. Rivera, R., \& Paradise, A. (2006). State of the industry: Trends in workplace learning and performance. Alexandria, VA: American Society for Training and Development.

Robie, B. D. (1999). Corporate Universities: Building Competences for Corporate Success'. Loma Resources. http://www.loma.org/res URL last visited 01/03/02018.

Ruggles, R. (1998). The state of the notion: knowledge management practice, California Management Review, Vol. 40 No. 3, pp. 80-9.

Scarborough, H., Swan, J. and Preston, J. (1999). Knowledge Management: A Literature Review, IPD, London. 
Schwandt, D.R. and Marquardt, M.J. (1999). Organizational Learning, St Lucie Press, Boca Raton, FL.

Scott, C. (2006). Challenging Google, Microsoft Unveils a Search Tool for Scholarly Articles. Chronicle of Higher Education. 52 (33): A43.

Shaw, S. (2005). The corporate university: Global or local phenomenon? Journal of European Industrial Training, 29(1), 21-39.

Skyrme, D. and Amidon, D. (1997). "The knowledge agenda", Journal of Knowledge Management, Vol. 1 No. 1, pp. 27-37.

Starbuck, W. (1992). Learning by knowledge-intensive firms, Journal of Management Studies, Vol. 29 No. 6, pp. 713-40.

Steck H. (2003). Corporatization of the University: Seeking Conceptual Clarity, ANNALS, AAPSS, 585, January 2003.

Stewart, J. (1999), Employee Development London: FT Pitman.

Stewart, T.A. (1997). Intellectual Capital: The New Wealth of Organizations, Doubleday, New York, NY.

Storey, J. (2004). Leadership development through corporate universities. Training and Management Development Methods, 18(4), 41-49.

Stumpf, S. (1998). Corporate universities of the future, Career Development International, Vol. 3 No. 5, pp. 206-11.

Sycheva, S.M. (2008). Uslovija i faktory sozdanija korporativnogo universiteta Conditions and factors of creating a corporate university. Vestnik universiteta, 9(1), $31-37$.

Taylor, S. y Phillips, T. (2002). The Corporate University Challenge: Corporate Competitiveness, Learning and Knowledge. Report of the EFMD Corporate University Learning Group, EFMD, Eindhoven/Open University, Milton Keynes.

Thomas, D. (1999). Seminar on Corporate Business Schools. Henley Management College 28th October.

Thompson, G. (2000). Unfulfilled prophecy: The evolution of corporate colleges. Journal of Higher Education, 71(3), 322-341.

Viscaña P y Uri be E. (2014). Aportes de la universidad corporativa a la ventaja competitiva de las organizaciones en Colombia, Universidad de Medellín facultad ciencias económicas y administrativas especialización en gestión del talento humano y la productividad cohorte 33 Medellín Vives L; Huges, J.; y LLie-Cardoza, C. (2015). Universidades corporativas Alineando Personas y Estrategias, Harvard Deusto Business Review, España.

Walton, J. (1999). Strategic Human Resource Development, London: Pitman.

Walton, J. (1999), Strategic Human Resource Development, Pearson Education, HarlowYoung, C.; Tuttle, R. (1969). The years 1919-1969 a history of General Motors Institute, General Motors Institute, 1969.

\footnotetext{
"Visión de Futuro" Año 16, Volumen No 23 N¹, Enero - Junio 2019 - Pág. 1 - 23

URL de la Revista: http://revistacientifica.fce.unam.edu.ar/

URL del Documento: http://revistacientifica.fce.unam.edu.ar/index.php?option=com content\&view=article\&id=492\&Itemid=101

ISSN 1668 - 8708 - Versión en Línea

E-mail: revistacientifica@fce.unam.edu.ar
} 


\section{RESUMEN BIOGRÁFICO}

\section{Manuel Alfonso Garzón Castrillon}

Pósdoutorado em Universidade de São Paulo, Brasil; PhD MSc. Profesional en Administración de Empresas, Actualmente Director del grupo de Investigación FIDEE, Email:manuelalfonsogarzon@fidee.org, Intereses actuales: Capacidades dinámicas; Gestión de la diversidad, Gestión de la felicidad; Gestión del conocimiento. ORCID ID http://orcid.org/0000- 0001-9009-3324. 